\title{
Local hole doping concentration modulation on graphene probed by tip-enhanced Raman spectroscopy
}

Takuya Iwasaki ${ }^{\mathrm{a}, \mathrm{b}, *}$, Taharh Zelai ${ }^{\mathrm{b}}$, Sheng $\mathrm{Ye}^{\mathrm{b}}$, Yoshishige Tsuchiya ${ }^{\mathrm{b}}$, Harold M. H. Chong ${ }^{\mathrm{b}}$, Hiroshi Mizuta ${ }^{\mathrm{a}, \mathrm{b}}$

School of Materials Science, Japan Advanced Institute of Science and Technology, 1-1 Asahidai, Nomi, Ishikawa 923-1292, Japan

Nanoelectronics and Nanotechnology Research Group, Faculty of Physical Sciences and Engineering, University of Southampton, Highfield, Southampton SO17 1BJ, UK

\begin{abstract}
We investigate local doping concentration modulation of graphene flakes on a $\mathrm{SiO}_{2} / \mathrm{Si}$ substrate that has been exposed to the same chemicals in device fabrication using tip-enhanced Raman spectroscopy (TERS). By spectral line scanning across the edge of graphene, it is observed that the D peak enhancement is localized in the vicinity of the edge boundary, and the TERS spatial resolution of $~ 228 \mathrm{~nm}$ is obtained. In the TERS spectra significant peak shifts of both the $G$ and 2D peaks are observed more than $7 \mathrm{~cm}^{-1}$ across the hump on graphene within the distance of $1 \mu \mathrm{m}$, while both $\mathrm{G}$ and $2 \mathrm{D}$ peaks are shifted less than $2 \mathrm{~cm}^{-1}$ in the far-field spectra. This indicates that the modulation of hole doping concentration in close proximity on graphene/SiO$/ 2 \mathrm{Si}$ can be
\end{abstract}

\footnotetext{
*Corresponding author. Tel: +81 761-51-1573. E-mail: t.iwasaki@jaist.ac.jp (Takuya Iwasaki)
} 
probed by using TERS surpassing the resolution of a laser diffraction limit of conventional micro Raman spectroscopy.

\section{INTRODUCTION}

Graphene is a two-dimensional material composed of $s p^{2}$ bonded carbon atoms with a hexagonal arrangement [1,2]. Because of its excellent mechanical and electrical properties, graphene has been expected for various applications, such as nanoelectromechanical systems [3], flexible thin film transistors [4], and radio frequency transistors [5]. It is known that electrical characteristics of graphene are influenced by defects [6], strain [7,8], and doping concentration modulation [9-12]. As those could be induced either intrinsically in the growth of graphene or extrinsically during the device fabrication process, a method to evaluate the quality of the graphene with high spatial resolution down to nanoscale must be very useful for detailed analysis of graphene nanoelectronic devices.

Raman spectroscopy has been extensively used as a non-destructive method to obtain various properties in graphene [13], such as the number of layers [14], edge [15], disorder [16], defect [6,17,18], strain [19-24], and doping [24-27]. However, conventional Raman spectroscopy only gives the averaged signal over the microscale laser spot size and its spatial resolution is limited by laser diffraction.

Tip-enhanced Raman spectroscopy (TERS) is a variant of Raman spectroscopy and is able to achieve better spatial resolution than conventional Raman spectroscopy via near-field effect by surface plasmon resonance [28]. Since the first TERS measurement for graphene in 2008 [29], TERS has been intensively used for investigating local material properties of various types of graphene, such as mechanically-exfoliated 
graphene on glass [30-38], on $\mathrm{Au}$ [39], chemical vapor deposition (CVD)-grown graphene on glass [38] or on $\mathrm{Cu}$ [39], epitaxially-grown graphene on $\mathrm{SiC}$ [40-42], and polymerized graphene [43]. On the other hand, there has been only little TERS work done for graphene nanodevice characterization.

In this study, we focus on using exfoliated graphene on $\mathrm{SiO}_{2} / \mathrm{Si}$ substrates because (1) extrinsic effects caused by device fabrication processes become more prominent thanks to its high crystal quality and (2) comparative study is possible by evaluating electrical characteristics of fabricated devices on the same platform at the same time. In the following, we demonstrate enhancement of the edge boundary signal on graphene on $\mathrm{SiO}_{2} / \mathrm{Si}$ and high TERS spatial resolution beyond laser diffraction limit. Significant peak shifts are observed in line-scanned TERS spectra taken across a hump structure on graphene, while only slight peak shifts are found in the far-field ones. The results suggest that TERS can pick up the modulation of local hole doping concentration with nanoscale resolution.

\section{EXPERIMENTAL}

Graphene flakes were mechanically exfoliated from a highly oriented pyrolytic graphite by using an adhesive tape and 290-nm-thick $\mathrm{SiO}_{2}$ layer grown on an n-doped Si substrate. The number of graphene layers was identified by the contrast between graphene and $\mathrm{SiO}_{2}$ in the optical image [44], and by micro Raman spectroscopy.

An optical image and Raman spectrum of a monolayer graphene are shown in Figs. 1(a) and (b). The number of graphene layers was identified by the contrast between graphene and $\mathrm{SiO}_{2}$ in the optical image and by fitting of the Raman peaks. The peak at $\sim 1590 \mathrm{~cm}^{-1}$, called $\mathrm{G}$ peak, is caused by the doubly-degenerated $E_{2 \mathrm{~g}}$ vibration mode at 
the Brillouin zone center in the first-order Raman scattering process. The D peak located $\sim 1345 \mathrm{~cm}^{-1}$ originates in the double resonance of the second-order process between different valleys around $\mathbf{K}$ and $\mathbf{K}^{\prime}$ in the Brillouin zone (intervalley scattering), which corresponds to the $A_{1 \mathrm{~g}}$ breathing mode at $\mathbf{K}$, the in-plane transverse optical phonon [16]. This peak is activated by defects, disorders or edges with armchair geometry $[15,45]$. The peak at $\sim 2685 \mathrm{~cm}^{-1}$ is the $2 \mathrm{D}$ peak (or called $\mathbf{G}^{\prime}$ peak), which arises from triple resonant process of the second-order intervalley scattering. Although the 2D peak corresponds to the overtone of the $\mathrm{D}$ peak, it is not required neither defects nor disorder for activation. We discriminate the number of graphene layers by fitting to the 2D peak, which has different profile shape with respect to the number of layers [14]. The 2D peak in Fig. 1(b) can be fitted with a single Lorentzian function whose full width at half maximum (FWHM) is $30.8 \pm 0.2 \mathrm{~cm}^{-1}$, indicating monolayer graphene [14].

Note that we have fabricated graphene nanoribbon devices on the same substrate but on the other flakes before all TERS measurements. The fabrication process is summarized in Fig. 1(c). Using a Poly(methyl methacrylate) (PMMA) resist, electron beam (EB) lithography and $\mathrm{O}_{2}$ plasma dry etching were performed to pattern nanoribbon structures. Metal contacts of $\mathrm{Cr} / \mathrm{Au}(2 / 40 \mathrm{~nm})$ were then fabricated by EB lithography with PMMA/MMA resists, EB deposition and lift-off processes. In consequence, graphene flakes we used in this study for TERS measurements on the same substrate were affected by this process so that they were no longer pristine. The topographic image of a fabricated device taken by atomic force microscopy (AFM) is shown in Fig. 1(d). The channel length and width are $\sim 3 \mu \mathrm{m}$ and $\sim 140 \mathrm{~nm}$, respectively. In electrical measurements, two-point resistance was measured by using a 
semiconductor device analyzer (Agilent B1500A) in an atmospheric environment at room temperature. Backgate modulation characteristics were measured by applying via the n-doped Si substrate under the constant source-drain bias voltage $V_{\mathrm{ds}}=10 \mathrm{mV}$.
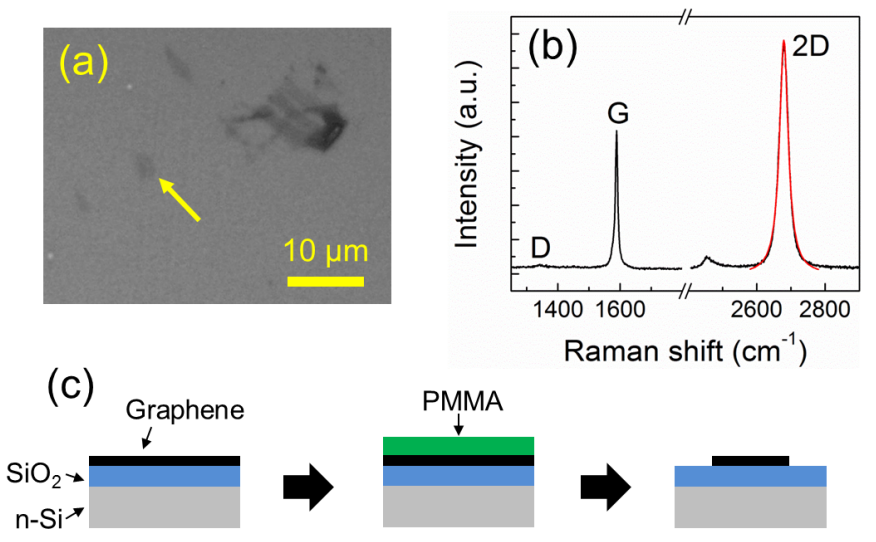

1. Exfoliation

2. Resist coating

3. EB lithography and Dry etching

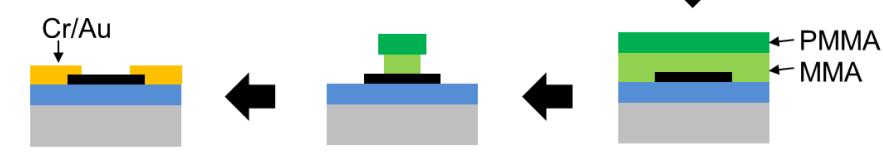

6. EB deposition

5. EB lithography 4. Resist coating
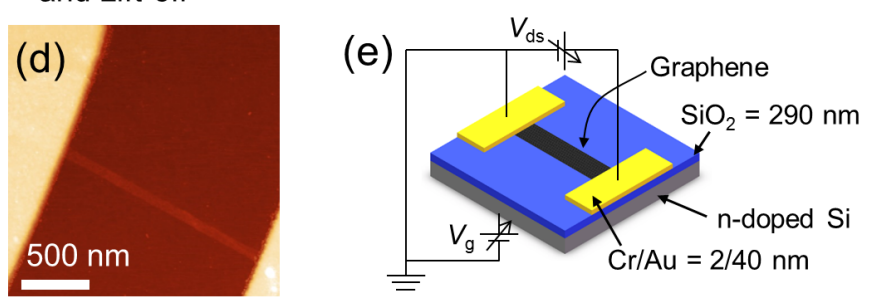

Fig. 1 - (a) The optical image of the monolayer graphene flake investigated in the this study. The yellow arrow points to the location of the flake. (b) The Raman spectrum of the monolayer graphene flake. The black line is experimental data, and the red line on the 2D peak is the fitted Lorentzian curve. (c) The device fabrication process flow. (d) The AFM topographic image of the graphene nanoribbon device on the same substrate. (e) The electrical measurement configuration for graphene devices. 
For the TERS measurements, we used the integrated system comprised of the Nanonics Multiview 4000 AFM system and Renishaw InVia Raman spectrometer. As shown in Fig. 2(a), the sample is exposed to laser from the top (top-illumination configuration), and the Raman spectrum is collected through the same objective lens (50× magnification, $\mathrm{NA}=0.45$ ). The Nd-doped Y-Al-garnet laser with the wavelength $\lambda$ of $532 \mathrm{~nm}$ was used for excitation. For feedback on AFM imaging and near-field excitation, a quartz tuning fork with a commercial probe tip was used (Nanonics Imaging Ltd.). This tip is coated with $\mathrm{Au}$, and the diameter of an Au nanoparticle on top of the tip is about $200 \mathrm{~nm}$. The laser power at the sample was kept less than $0.4 \mathrm{~mW}$ to avoid damage to the graphene and tip by heating. During scanning measurements, the in-plane position $(\mathrm{x}, \mathrm{y})$ of the sample was controlled by the sample piezo stage, and the tip height (z) was controlled by the tuning fork feedback, so as not to misalign the laser spot and the tip location. The first Raman spectrum at the selected point was taken when the tip was approached and then second one when retracted from the sample with the distance between tip and sample of $\sim 10 \mu \mathrm{m}$. The spectrum with the tip approached contains both near-field and far-field effects, while the one with the tip retracted only the far-field effect. The result of the near-field effect was obtained by subtracting the tip-retracted spectrum from the tip-approached one. Figure 2(b) exhibits tip-approached and retracted spectra at the same point on the monolayer graphene flake. The enhancement of each peak has been confirmed in the subtracted spectrum shown in Fig. 2(c). 

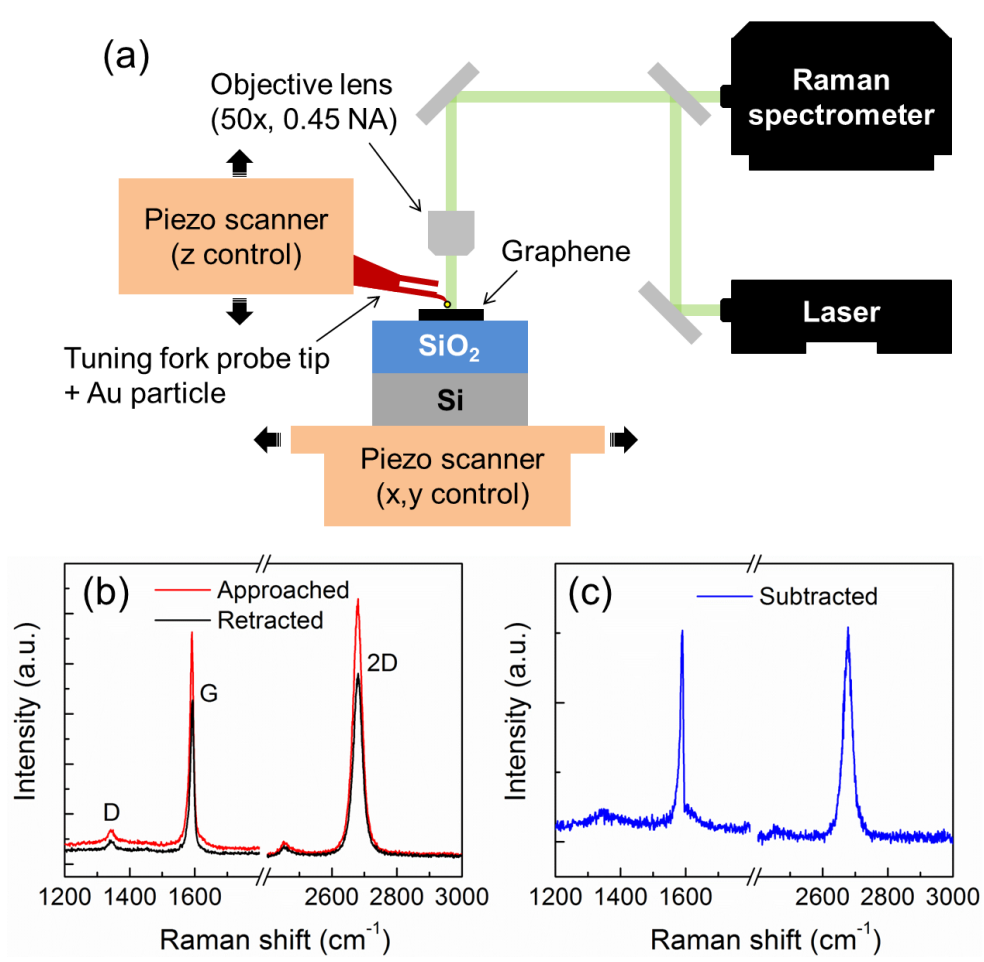

Fig. 2 - (a) The schematic of the TERS measurement setup with the top-illumination configuration. (b) The Raman spectra of the monolayer graphene by the TERS measurement. The red and black lines represent the spectrum acquired when the tip is approached and retracted, respectively. (c) The subtracted Raman spectrum shown in (b) (i.e., Approached - Retracted).

\section{RESULTS}

\subsection{Electrical measurements}

First, electrical characteristics for the graphene nanoribbon transistors were measured to evaluate the doping concentration of the graphene via the position of the charge neutrality point (CNP) in resistance vs backgate voltage $V_{\mathrm{g}}$ curves. The CNP is the point where the electron and the hole density become same, a shift of the CNP to the positive (negative) in $V_{\mathrm{g}}$ means that Fermi energy in graphene moves far in the valence 
(conduction) band, i.e., hole (electron) doping concentration is enhanced. Figure 3 shows the measured resistance as a function of $V_{\mathrm{g}}$, and the CNP appears in the positive $V_{\mathrm{g}}$ range. The carrier density $n$ is estimated by the following equation;

$$
n=\frac{C_{\mathrm{g}} V_{\mathrm{CNP}}}{e}
$$

where $C_{\mathrm{g}}$ is the gate capacitance per area, $V_{\mathrm{CNP}}$ is the gate voltage at the CNP, and $e$ is the elemental charge $[1,9,10]$. Based on the parallel plate capacitor model, $C_{\mathrm{g}}$ is calculated to be $\sim 11.9 \mathrm{nF} \mathrm{cm}{ }^{-2}$ for the dielectric $\mathrm{SiO}_{2}$ of $290 \mathrm{~nm}$. Then, the doped hole density is estimated to be $\sim 2.32 \times 10^{12} \mathrm{~cm}^{-2}$. Note that, all 15 devices fabricated on the same substrate show $V_{\mathrm{CNP}}>0 \mathrm{~V}$ suggesting our device fabrication process is ended up with hole doping.

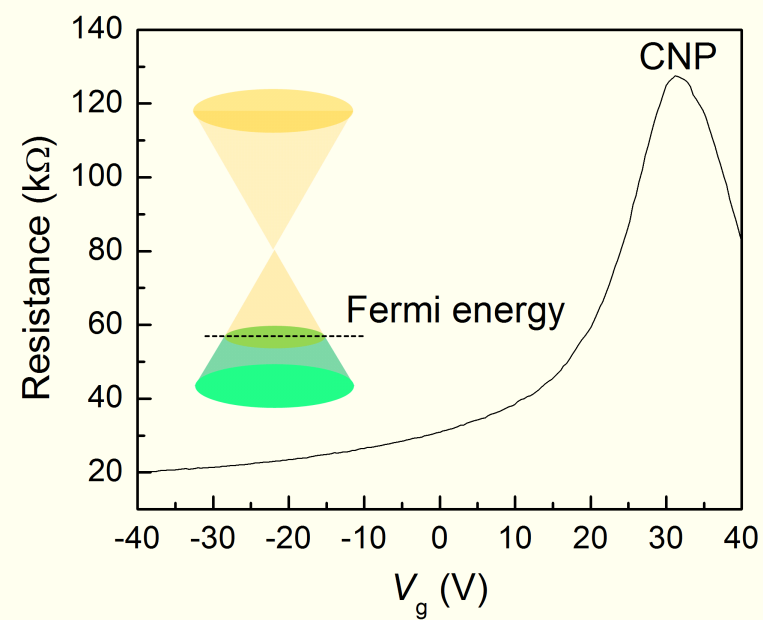

Fig. 3 - The resistance of the graphene nanoribbon transistor as a function of $V_{\mathrm{g}}$ at room temperature. The maximum resistance appears at the CNP of $V_{\mathrm{g}}=31.2 \mathrm{~V}$. The inset illustrates the band structure of the hole-doped graphene with Fermi energy at the valence band. 


\subsection{TERS scanning across the edge boundary}

Figure 4(a) shows an AFM topographic image of a monolayer graphene flake on the same substrate where the nanoribbon devices are fabricated. Spectral line scanning is performed across the edge of the flake along the dotted arrow drawn in Fig. 4(a). At the outside of the graphene flake marked by the cross mark shown in Fig. 4(a), both D and G peaks have not been enhanced, so the subtracted spectrum does not show any peaks (Fig. 4(b)). In contrast, both of peaks are enhanced at the edge marked by the circle in Fig. 4(c). At the triangle mark graphene region, the $G$ peak is enhanced while the D peak not as shown in Fig. 4(d). In the height profile along the scanning line shown in Fig. 4(e), the edge of graphene is identified at the position $x \sim 1000 \mathrm{~nm}$. The G peak intensity starts to increase around the edge boundary, and remain constant when $x>1000 \mathrm{~nm}$ (Fig. 4(f)). The D peak enhancement is observed only around the edge boundary of $x \sim 1000 \mathrm{~nm}$ as shown in Fig. 4(g). From the G peak intensity profile, the TERS spatial resolution is extracted using the following equation [33];

$$
\begin{aligned}
I_{\mathrm{G}}=\sqrt{\frac{\pi}{8}} A_{1} w_{1} & {\left[1-\operatorname{erf}\left\{\frac{\sqrt{2}\left(-x+x_{\text {edge }}\right)}{w_{1}}\right\}\right] } \\
& +\sqrt{\frac{\pi}{8}} A_{2} w_{2}\left[1-\operatorname{erf}\left\{\frac{\sqrt{2}\left(-x+x_{\text {edge }}\right)}{w_{2}}\right\}\right]
\end{aligned}
$$

where $I_{\mathrm{G}}$ is the $\mathrm{G}$ peak intensity in the subtracted spectrum, $A_{1}$ and $A_{2}$ are scaling factors, $x_{\text {edge }}$ is the position of edge, $w_{1}$ and $w_{2}$ are the spatial resolution of far-field and near-field spectra, respectively. The far-field resolution is calculated based on the laser spot size to be $w_{1} \cong 1.22 \lambda / \mathrm{NA} \sim 1.44 \mu \mathrm{m}$, and then, the TERS resolution is extracted to be $w_{2} \sim 228 \mathrm{~nm}$, which is comparable to the Au particle size at the tip. The D peak intensity profile can be fitted by a Gaussian curve with the FWHM of 268 nm. 

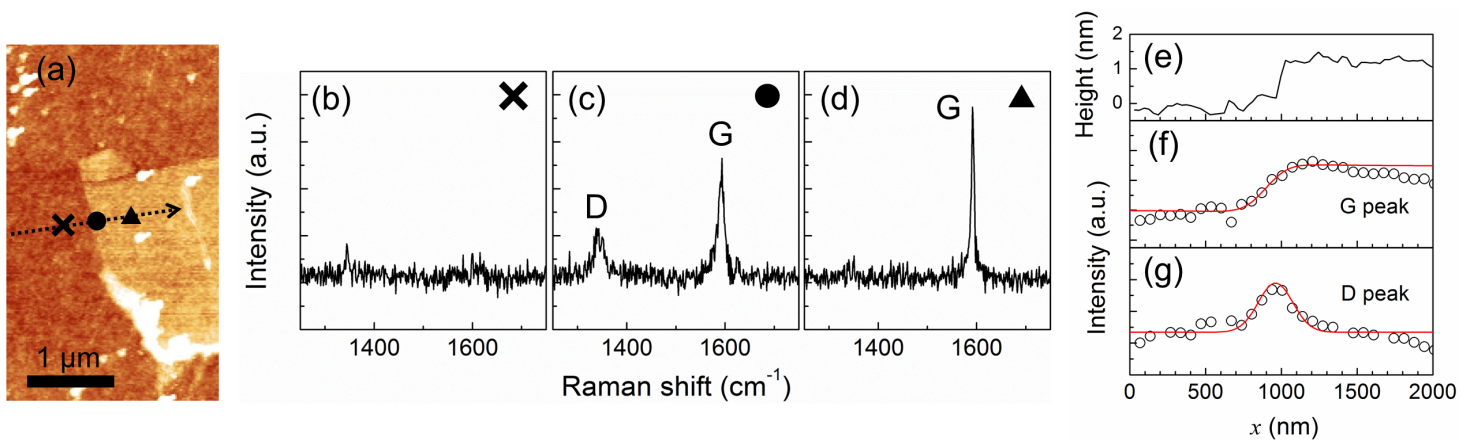

Fig. 4 - (a) The AFM image of the monolayer graphene flake. The spectral line scanning is performed along the dotted arrow. (b) The subtracted Raman spectra acquired at the cross (outside of the graphene flake), (c) the circle (edge), (d) and the triangle (inside) marks (shown in (a)), respectively. (e) The AFM height profile, (f) the intensity profile of the subtracted spectra for the G peaks and (g) the D peaks along the arrow shown in (a). The tail of the arrow corresponds to $x=0 \mathrm{~nm}$. The red lines in (f) and (g) are fitting curves using the equation (2) and the Gaussian function, respectively.

\subsection{Raman peak variation inside graphene}

We have also conducted spectral line scanning inside the graphene flake along the arrow shown in Fig. 5(a). In the topological profile along the arrow in Fig. 5, a hump is identified at $x \sim 950 \mathrm{~nm}$. Line shapes of the G peak in far-field Raman and in TERS taken at five different positions on the line are plotted in Figs. 5(c) and (d), respectively. There is almost no change in the far-field Raman spectra, whereas the shift of the peak positions is clearly identified in TERS. Clear difference between the far-field and tip-enhanced Raman spectra is also observed in monitoring the 2D peak as shown in Figs. 5(e) and (f). The peak center and FWHM of each peak are extracted using single Lorentzian curve fitting and plotted with respect to the position along the line in Figs. 5(g)-(j). For the far-field spectra, overall peak shift of the G and 2D peaks is less than 
$2 \mathrm{~cm}^{-1}$ in the whole range of $x$. On the other hand, the G peak center in TERS is $1590.47 \pm 0.28 \mathrm{~cm}^{-1}$ at $x \sim 0 \mathrm{~nm}$ and then shifted to $1598.14 \pm 0.23 \mathrm{~cm}^{-1}$ at $x=950 \mathrm{~nm}$. The 2D peak center is observed to be $2675.26 \pm 1.09 \mathrm{~cm}^{-1}$ at $x=0 \mathrm{~nm}$, and then, shifted to $2682.32 \pm 0.25 \mathrm{~cm}^{-1}$ at $x=950 \mathrm{~nm}$. For the FWHM, again almost no change in the far-field spectra whereas the FWHM of the G peak is changed from $20 \mathrm{~cm}^{-1}$ at around $x=300 \sim 800 \mathrm{~nm}$ to $15.69 \pm 0.75 \mathrm{~cm}^{-1}$ at $x=950 \mathrm{~nm}$. The 2D peak FWHM is $\sim 30 \mathrm{~cm}^{-1}$ around $x \sim 100 \mathrm{~nm}$, that broadens to be $41.56 \pm 1.04 \mathrm{~cm}^{-1}$ at $x=950 \mathrm{~nm}$. 

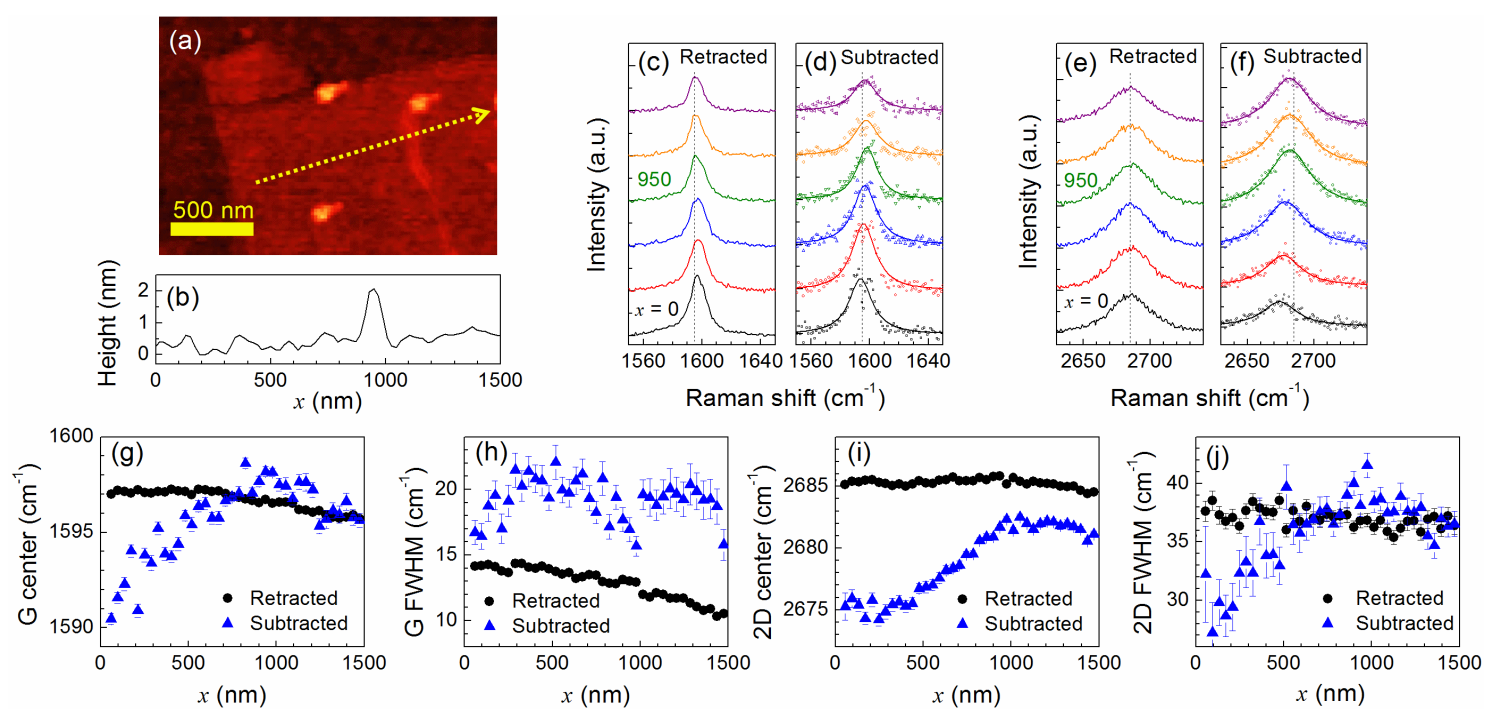

Fig. 5 - (a) The AFM image of the graphene flake. The spectral line scanning is performed along the yellow dotted arrow. (b) The AFM height profile along the arrow shown in (a). The tail of the arrow corresponds to $x=0 \mathrm{~nm}$. (c)-(f) The tip-retracted Raman spectra and the subtracted one around the G peak (c),(d) and the 2D peak (e),(f), respectively, at each position along the arrow shown in (a). The subtracted data (symbols) are fitted with the Lorentzian curve (solid line). Each plot has the offset to be seen clearly. The black and green plots correspond to the spectrum at $x=0 \mathrm{~nm}$ and $950 \mathrm{~nm}$, respectively. The vertical dotted lines in (c),(d) and (e),(f) point to $1595 \mathrm{~cm}^{-1}$ and $2685 \mathrm{~cm}^{-1}$, respectively, for eye guides. (g),(h) The center and the FWHM of the G peak along the arrow shown in (a), respectively. (i),(j) The center and the FWHM of the 2D peak along the arrow shown in (a), respectively. In (g)-(j), the black and blue color plots are the results of the Lorentzian function fitting to the tip-retracted and the subtracted data, respectively. The error bars indicate fitting error.

\section{DISCUSSION}

First we briefly discuss the results of the electrical measurements that suggest all the 
graphene on the same substrate are hole-doped. Considering the transfer process and the fact that the flakes are directly placed on $\mathrm{SiO}_{2}$, this hole doping can be attributed to chemical charge transfer from physisorbed $\mathrm{O}_{2}, \mathrm{H}_{2} \mathrm{O}$ molecules, and polymer residues on the graphene surface [9-11].

Next we discuss the results of the line scanning across the edge. As shown in Fig. 4(f), the $G$ peak is enhanced from the edge boundary and remained constant beyond. Taking account of the origin of the $G$ peak that corresponds to the $E_{2 \mathrm{~g}}$ mode, its intensity would increase with increasing the number of excited $s p^{2}$ bonded carbon rings within the laser spot. Thanks to the near-field effect the increase of the intensity of $G$ peak in the vicinity of the edge is clearly observed. The intensity does not largely change inside the graphene flake because the exposed area is constant. On the other hand, the D peak intensity is enhanced only around $x \sim 1000 \mathrm{~nm}$. This indicates that the D mode is strongly localized in the vicinity of the edge, which is consistent with the previous reports $[33,35]$. Actually in $x<1000 \mathrm{~nm}$, the $\mathrm{D}$ and the $\mathrm{G}$ peaks are observed both in the far-field and tip-approached Raman spectra as the laser spot $(\sim 1 \mu \mathrm{m})$ is overlapped with the graphene region. However, the spectra are hardly enhanced in $x<1000 \mathrm{~nm}$ as shown in Fig. 4(b), implying that the near-field spot does not reach to the graphene region. We have obtained two comparable spatial resolutions by two different two ways by curve fitting to the $\mathrm{G}$ peak and the D peak intensity profile. Clearly both spatial resolutions of our TERS measurements are over the diffraction limit of light. The extracted values are mainly due to the size of the Au nanoparticle on top of the tip. The message from this analysis is that TERS is also applicable for device structure analysis with a good level of the spatial resolution which is comparable to the previous TERS studies for materials. The results of line scanning inside the graphene are next discussed by focusing on 
significant peak variation observed in the TERS spectra. In normal Raman spectroscopy, such behavior could be explained by considering (i) doping and (ii) strain effects. Doping causes an up-shift for the G peak, and the G peak FWHM narrows as increasing both carrier doping as a result of the nonadiabatic Kohn anomaly [25,26,46]. The 2D peak down-shifts or up-shifts by electron or hole doping, respectively, due to charge transfer [26], and the 2D peak FWHM broadens as increasing doping [27]. Strain effects can be discriminated either uniaxial or biaxial, and then, tensile and compressive strain. Uniaxial strain splits the $G$ peak into $G^{-}$and $G^{+}$with down-shifting, and it splits or broadens the 2D peak [21,22], while biaxial one does not affect the FWHM of both G and 2D peaks [23]. Tensile strain causes down-shift due to phonon softening, and opposite for compressive one owing to phonon stiffening $[13,19,20]$. According to Ref. 24 , the ratio of the $2 \mathrm{D}$ peak shift to the $\mathrm{G}$ peak shift is a good index to evaluate which effect is more dominant on the Raman shift. Our result of $\sim 0.87$ at $0<x<950 \mathrm{~nm}$ is much closer to 0.75 , a typical value for the case that the doping effects are dominant, than to 2.2 for strain effects. Therefore, it can be interpreted that the main contribution of peak shift observed within $x=0 \sim 950 \mathrm{~nm}$ is doping concentration modulation. Comparing the peak center at $x=950 \mathrm{~nm}$ to that at $x=0 \mathrm{~nm}$, the 2D peak up-shifts as approaching from $x=0 \mathrm{~nm}$ to $x=950 \mathrm{~nm}$ suggesting that the position $x=950 \mathrm{~nm}$ has higher hole doping concentration than $x=0 \mathrm{~nm}$. As is shown in the AFM image and the height profile (Figs. 4(a), (b)), a hump structure with $~ 1.5 \mathrm{~nm}$ in height and $100 \mathrm{~nm}$ in width is clearly observed around $x=950 \mathrm{~nm}$, where the enhancement of hole doping concentration is suggested by the analysis of TERS spectra. Here we discuss how this hump structure is associated with the local doping concentration modulation. One possible explanation is that this hump structure could attract molecules to adsorb onto 
its surface due to the larger effective surface area than the flat region, and the adsorbates come from an ambient environment, such as moisture and $\mathrm{O}_{2}$ molecules acting as acceptors for graphene $[9,10]$. In addition, use of solvent and water during fabrication can intercalate inside the wrinkle (i.e., between graphene and $\mathrm{SiO}_{2}$ ), resulting in inducing hole doping into graphene [47]. In contrast, the strain caused by this structure is considered very small because the aspect ratio of the height to the width is significantly small of $\sim 0.015$. The small peak shift observed in the far-field spectra is interpreted as that local doping is averaged within $\sim 1 \mu \mathrm{m}$ of the entire laser spot. Note that, Ref. 25 mentioned that the scale of charge inhomogeneity is less than $1 \mu \mathrm{m}$, which also agrees with our interpretation.

\section{CONCLUSION}

We have investigated the modulation of local hole doping concentration in graphene supported on the $\mathrm{SiO}_{2} / \mathrm{Si}$ substrate by using TERS. In the electrical measurements of the graphene nanoribbon transistors, we confirmed heavily hole doping in almost graphene on the same substrate. By spectral line scanning across the edge of graphene, we observed the localization of the $\mathrm{D}$ peak enhancement in the vicinity of the edge boundary, and the TERS spatial resolution of $\sim 228 \mathrm{~nm}$ was obtained. The TERS results of being scanned inside the graphene flake which experienced the device fabrication process also show the distinct peak shift at different positions within $\sim 1 \mu \mathrm{m}$ range, thus

resolving the local doping concentration modulation with nanoscale resolution. Even though the fabrication technology has been highly developed, it is inevitable that unintentional, distortion and contaminations can contribute to the doping effect through microfabrication processes $[24,27]$. TERS scanning measurements can show the critical 
position of process-induced disorder, strain, and doping concentration, and hence, one can select the region with the ideal quality of graphene for device fabrication. Our study demonstrates a way to analyze the local properties associated with Raman spectroscopy in nanoscale on graphene devices based on graphene/SiO $2 / \mathrm{Si}$ architectures and also on opaque substrates.

\section{ACKNOWLEDGMENT}

This work is supported by a Grant-in-Aid for Scientific Research No. 25220904 and Grant-in-Aid for JSPS Fellows from the Japan Society for the Promotion of Science, by the Center of Innovation Program of the Japan Science and Technology Agency, by Southampton Nanofabrication Centre, and by the SOU-JAIST Collaborative Education and Research Co-supervision Programme from Univ. of Southampton and JAIST. The authors would like to thank Nanonics Imaging Ltd. for their TERS measurement advice and probe tips.

\section{REFERENCES}

[1] Novoselov KS, Geim AK, Morozov SV, Jiang D, Zhang Y, Dubonos SV, et al. Electric field effect in atomically thin carbon films. Science 2004;306(5969):666-9.

[2] Geim AK, Novoselov KS. The rise of graphene. Nat Mater 2007;6:183-91.

[3] Sun J, Schmidt ME, Manoharan M, Chong HMH, Mizuta H. Large-scale nanoelectromechanical switches based on directly deposited nanocrystalline graphene on insulating substrates. Nanoscale 2016;8(12):6659-65.

[4] Akinwande D, Petrone N, Hone J. Two-dimensional flexible nanoelectronics. Nat Commun 2014;5:5678. 
[5] Schwierz F. Graphene transistors. Nat Nanotech. 2010;5:487-96.

[6] Moktadir Z, Hang S, Mizuta H, Defect-induced Fermi level pinning and suppression of ambipolar behaviour in graphene. Carbon 2015;93:325-34.

[7] Teague ML, Lai AP, Velasco J, Hughes CR, Beyer AD, Bockrath MW, et al. Evidence for strain-induced local conductance modulations in single-layer graphene on $\mathrm{SiO}_{2}$. Nano Lett 2009;9(7):2542-6.

[8] Lee Y, Bae S, Jang H, Jang S, Zhu SE, Sim SH, et al. Wafer-scale synthesis and transfer of graphene films. Nano Lett 2010;10(2):490-3.

[9] Schedin F, Geim AK, Morozov SV, Hill EW, Blake P, Katsnelson MI, et al. Detection of individual gas molecules adsorbed on graphene. Nat Mater 2007;6:652-5. [10] Sato Y, Takai K, Enoki T. Electrically controlled adsorption of oxygen in bilayer graphene devices. Nano Lett 2011;11(8):3468-75.

[11] Pirkle A, Chan J, Venugopal A, Hinojos D, Magnuson CW, McDonnell S, et al. The effect of chemical residues on the physical and electrical properties of chemical vapor deposited graphene transferred to $\mathrm{SiO}_{2}$. Appl Phys Lett 2011;99(12):122108.

[12] Chen JH, Jang C, Adam S, Fuhrer MS, Williams ED, Ishigami M. Charged-impurity scattering in graphene. Nat Phys 2008;4:377-81.

[13] Ferrari AC, Basko DM. Raman spectroscopy as a versatile tool for studying the properties of graphene. Nat Nanotech 2013;8:235-46.

[14] Ferrari AC, Meyer JC, Scardaci V, Casiraghi C, Lazzeri M, Mauri F, et al. Raman spectrum of graphene and graphene layers. Phys Rev Lett 2006;97(18):187401.

[15] You Y, Ni Z, Yu T, Shena Z. Edge chirality determination of graphene by Raman spectroscopy. Appl Phys Lett 2008;93(16):163112.

[16] Ferrari AC. Raman spectroscopy of graphene and graphite: disorder, 
electron-phonon coupling, doping and nonadiabatic effects. Solid State Commun 2007;143(1-2):47-57.

[17] Hang S, Moktadir Z, Mizuta H. Raman study of damage extent in graphene nanostructures carved by high energy helium ion beam. Carbon 2014;72:233-41.

[18] Eckmann A, Felten A, Mishchenko A, Britnell L, Krupke R, Novoselov KS, et al. Probing the nature of defects in graphene by Raman spectroscopy. Nano Lett 2012;12(8):3925-30.

[19] Mohiuddin TMG, Lombardo A, Nair RR, Bonetti A, Savini G, Jalil R, et al. Uniaxial strain in graphene by Raman spectroscopy: G peak splitting, Grüneisen parameters, and sample orientation. Phys Rev B 2009;79(20):205433.

[20] Larsen MBBS, Mackenzie DMA, Caridad JM, Bøggild P, Booth TJ. Transfer induced compressive strain in graphene: evidence from Raman spectroscopic mapping. Microelectronic Eng 2014;121:113-7.

[21] Huang M, Yan H, Heinz TF, Hone J. Probing strain-induced electronic structure change in graphene by Raman spectroscopy. Nano Lett 2010;10(10):4074-9.

[22] Frank O, Mohr M, Maultzsch J, Thomsen C, Riaz I, Jalil R, et al. Raman 2D-band splitting in graphene: theory and experiment. ACS Nano 2011;5(3):2231-9.

[23] Zabel J, Nair RR, Ott A, Georgiou T, Geim AK, Novoselov KS, et al. Raman spectroscopy of graphene and bilayer under biaxial strain: bubbles and balloons. Nano Lett 2012;12(2):617-21.

[24] Lee JE, Ahn G, Shim J, Lee YS, Ryu S. Optical separation of mechanical strain from charge doping in graphene. Nat Commun 2012;3:1024.

[25] Casiraghi C, Pisana S, Novoselov KS, Geim AK, Ferrari AC. Raman fingerprint of charged impurities in graphene. Appl Phys Lett 2007;91(23):233108. 
[26] Das A, Pisana S, Chakraborty B, Piscanec S, Saha SK, Waghmare UV. Monitoring dopants by Raman scattering in an electrochemically top-gated graphene transistor. Nat Nanotech 2008;3:210-5.

[27] Wang QH, Jin Z, Kim KK, Hilmer AJ, Paulus GLC, Shih CJ, et al. Understanding and controlling the substrate effect on graphene electron-transfer chemistry via reactivity imprint lithography. Nat Chem 2012;4:724-32.

[28] Stadler J, Schmid T, Zenobi R. Developments in and practical guidelines for tip-enhanced Raman spectroscopy. Nanoscale 2012;4(6):1856-70.

[29] Hoffmann GG, With G de, Loos J. Micro-Raman and tip-enhanced Raman spectroscopy of carbon allotropes. Macromol Symp 2008;265(1):1-11.

[30] Saito Y, Verma P, Masui K, Inouye Y, Kawata S. Nano-scale analysis of graphene layers by tip-enhanced near-field Raman spectroscopy. J Raman Spectrosc 2009;40(10):1434-40.

[31] Snitka V, Rodrigues RD, Lendraitis V. Novel gold cantilever for nano-Raman spectroscopy of graphene. Microelectronic Eng 2011;88(8):2759-62.

[32] Ikeda K, Takase M, Hayazawa N, Kawata S, Murakoshi K, Uosaki K. Plasmonically nanoconfined light probing invisible phonon modes in defect-free graphene. J Am Chem Soc 2013;135(31):11489-92.

[33] Su W, Roy D. Visualizing graphene edges using tip-enhanced Raman spectroscopy. J Vac Sci Technol B 2013;31(4):041808.

[34] Rickman RH, Dunstan PR. Enhancement of lattice defect signatures in graphene and ultrathin graphite using tip-enhanced Raman spectroscopy. J Raman Spectrosc 2014;45(1):15-21.

[35] Beams R, Cancado LG, Oh SH, Jorio A, Novotny L. Spatial coherence in near-field 
Raman scattering. Phys Rev Lett 2014;113(18):186101.

[36] Beams R, Cancado LG, Jorio A, Vamivakas AN, Novotny L. Tip-enhanced Raman mapping of local strain in graphene. Nanotechnology 2015;26(17):175702.

[37] Pashaee F, Sharifi F, Fanchini G, Labarthet FL. Tip-enhanced Raman spectroscopy of graphene-like and graphitic platelets on ultraflat gold nanoplates of $20 \mathrm{~nm}$. Phys Chem Chem Phys 2015;17(33):21315-22.

[38] Ghislandi M, Hoffmann GG, Tkalya E, Xue L, De With G. Tip-enhanced Raman spectroscopy and mapping of graphene sheets. Appl Spectrosc Rev 2012;47(5):371-81.

[39] Stadler J, Schmid T, Zenobi R. Nanoscale chemical imaging of single-layer graphene. ACS Nano 2011;5(10):8842-8.

[40] Domke KF, Pettinger B. Tip-enhanced Raman spectroscopy of 6H-SiC with graphene adlayers: selective suppression of E1 modes. J Raman Spectrosc 2009;40(11):1427-33.

[41] Vantasin S, Tanabe I, Tanaka Y, Itoh T, Suzuki T, Kutsuma Y, et al. Tip-enhanced Raman scattering of the local nanostructure of epitaxial graphene grown on $4 \mathrm{H}-\mathrm{SiC}$ (000-1). J Phys Chem C 2014;118(44):25809-15.

[42] Suzuki T, Itoh T, Vantasin S, Minami S, Kutsuma Y, Ashida K, et al. Tip-enhanced Raman spectroscopic measurement of stress change in the local domain of epitaxial graphene on the carbon face of $4 \mathrm{H}-\mathrm{SiC}(000-1)$. Phys Chem Chem Phys 2014;16(37):20236-40.

[43] Shiotari A, Kumagai T, Wolf M. Tip-enhanced Raman spectroscopy of graphene nanoribbons on Au(111). J Phys Chem C 2014;118(22):11806-12.

[44] Blake P, Hill EW, Castro Neto AH, Novoselov KS, Jiang D, Yang R, et al. Making graphene visible. Appl Phys Lett 2007;91(6):063124. 
[45] Thomsen C, Reich S. Double resonant Raman scattering in graphite. Phys Rev Lett 2000;85(24):5214-7.

[46] Lazzeri M, Mauri F. Nonadiabatic Kohn anomaly in a doped graphene monolayer. Phys Rev Lett 2006;97(26):266407.

[47] Kim HH, Yang JW, Jo SB, Kang B, Lee SK, Bong H, et al. Substrate-induced solvent intercalation for stable graphene doping. ACS Nano 2013;7(2):1155-62. 


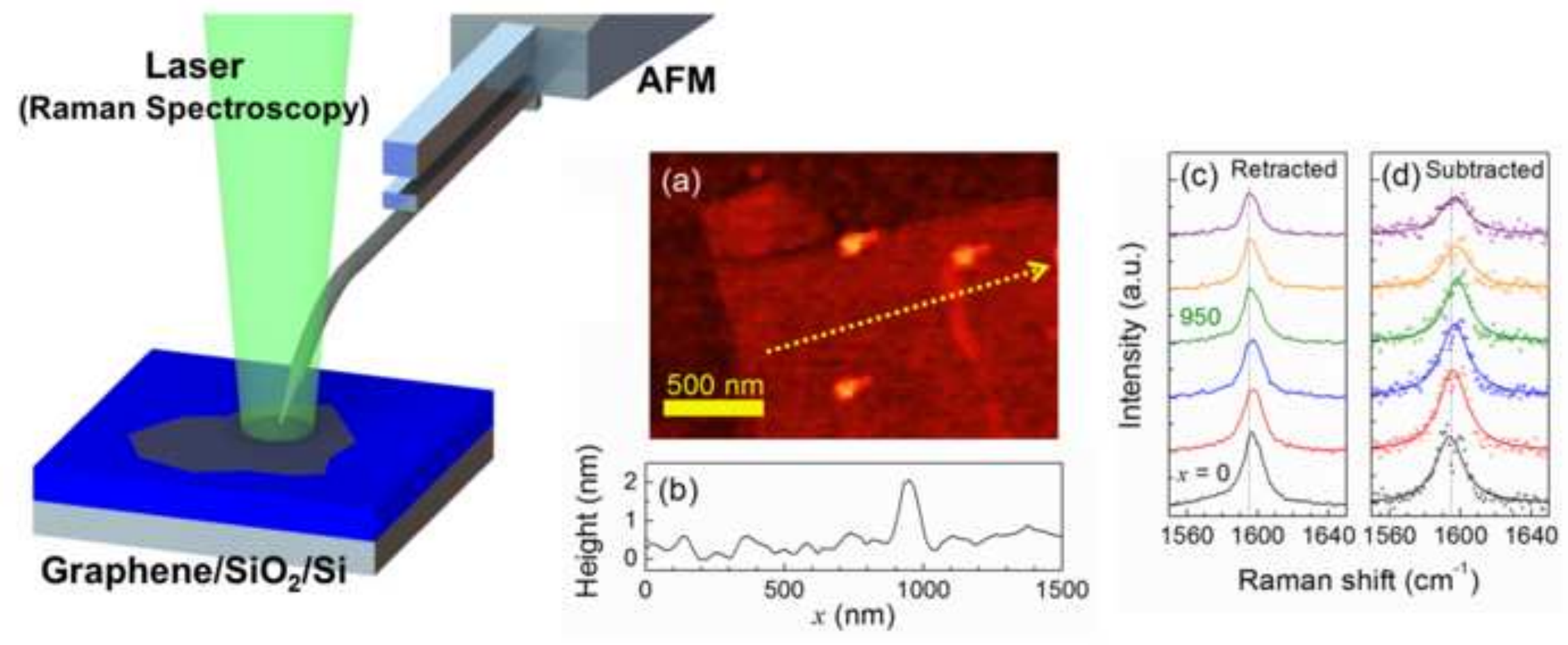

